\title{
Reverse Shoulder Arthroplasty Versus Antegrade Nailing for 3- and 4-Part Proximal Humeral Fractures in Elderly Patients.
}

\section{Khalil AMRI}

Military Hospital of Tunis

Rabie AYARI ( $\square$ rabie.ayari@hotmail.com )

Military Hospital of Tunis https://orcid.org/0000-0001-5949-0022

Karim LATRECH TLEMSANI

Military Hospital of Tunis

Achraf ABDENNADHER

Military Hospital of Tunis

Mohamed BEN SALAH

Charles-Nicolle Hospital Tunis

Rim DHAHRI

Military Hospital of Tunis

Chadia CHOURABI

Military Hospital of Tunis

\section{Lotfi NOUISRI}

Military Hospital of Tunis

\section{Research article}

Keywords: Proximal humeral fractures, Intramedullary nailing, Reverse arthroplasty, Anatomical results, Functional results, Complications

Posted Date: September 10th, 2020

DOl: https://doi.org/10.21203/rs.3.rs-73500/v1

License: (c) (i) This work is licensed under a Creative Commons Attribution 4.0 International License. Read Full License 


\section{Abstract}

\section{Introduction:}

Proximal humerus fractures are prevalent and widespread, accounting for $5 \%$ of all fractures, mainly in the elderly osteoporotic patients following a low-level trauma. Three-and four-part fractures represent 13 to $16 \%$ of these fractures and are generally associated with a worse prognosis. Depending on the fracture type and the patient status, several therapeutic options would be available ranging from osteosynthesis to arthroplasty.

The aim of our study was to compare the functional and radiological results, as well as the complications of reverse shoulder arthroplasty and anterograde nailing when managing these fractures.

\section{Methods:}

A retrospective and descriptive study was conducted within the orthopedic surgery department of the Military Hospital of Instruction of Tunis, over a period of 42 months, from January 2016 to June 2019 with an average follow-up of 18 months.

We included 31 cases of complex fractures of the proximal humerus in elderly patients, aged over 65 years, 16 of whom were treated by reverse total arthroplasty and 15 by antegrade nailing.

\section{Results:}

Our patient pool included 8 men and 23 women with a mean age of 73 years, ranging from 65 to 85 . A low-energy fall was the main cause of the fractures with the dominant side being affected in $55 \%$ of cases. Four-part fractures were predominant representing $68 \%$ of the fractures. Constant and DASH scores at the last follow-up were at 68 and 24 respectively. Most patients were satisfied with the outcome of their surgeries, with an overall satisfaction rate of $87 \%$. The complications rate was in accordance with the literature. Functional results in the group treated with reverse total shoulder arthroplasty were better with a lower complications rate.

\section{Conclusion:}

Reverse total shoulder arthroplasty has become an interesting surgical alternative, as the functional and anatomical results in our study were promising, while elucidating an improved quality of life for our patients. These findings have encouraged us to continue using this technique as a first-line choice for complex fractures of the proximal humerus in the elderly

\section{Introduction:}

Proximal humerus fractures are prevalent and widespread, accounting for $5 \%$ of all fractures, mainly in the elderly osteoporotic patients following a low-level trauma [1, 2]. Three-and four-part fractures 
represent 13 to $16 \%$ of these fractures and are generally associated with a worse prognosis given the higher risk of humeral head necrosis [3].

Depending on the fracture type and the patient status, several therapeutic options would be available ranging from osteosynthesis to arthroplasty. The decision can be difficult even for experienced surgeons.

Only few studies evaluated the results of 3- and 4-part fractures treated with open reduction internal fixation (ORIF). To our knowledge there is no study comparing the outcomes of intramedullary antegrade nailing and reverse shoulder arthroplasty (RSA) when managing these fractures.

The aim of our study was to compare the functional and radiological results, as well as the complications of these fractures managed by both methods, in elderly patients.

\section{Methods:}

A retrospective and descriptive study was conducted within the orthopedic surgery department of the Military Hospital of Instruction of Tunis, over a period of 42 months, from January 2016 to June 2019 with an average follow-up of 18 months.

We included 31 patients aged over 65 years old, who presented with recent, closed post traumatic 3- or 4part fractures of the proximal humerus.

The RSA group included 16 fractures whereas the nailing group included 15 fractures.

Minimal follow up was of 1 year. Exclusion criteria were age $<65$ years, insufficient follow-up (< 12 months), segmental fractures and polytraumas, pathological bone, and lack of appropriate postoperative radiographs.

All patients had comorbidities. $30 \%$ had a history of a cardiovascular disease.

Preoperative radiographs included a true anteroposterior (AP) and a lateral view as well as a CT scan.

We used the Neer's classification [4] to evaluate the fracture's pattern.

All surgeries were performed by one surgeon. We used "Arrow" reverse prostheses and "TumTum" anterograde humeral nails.

All patients underwent surgery under general anesthesia in the beach chair position. We used a superolateral approach exclusively. Care was taken to reduce the tuberosities. The incision was closed on a Redon drain, which was left for 48 hours. All patients were immobilized postoperatively for 4 weeks. Rehabilitation followed the unit protocol starting one day after surgery.

Patients underwent clinical and radiographic follow-up at 6 weeks, 3 months, 12 months and then yearly. Postoperative radiographs included a true AP (alpha angle evaluation) and a lateral view (gamma angle 
evaluation). Normal alpha angle values range from $30^{\circ}$ to $60^{\circ}$. Evaluation of tuberosities' healing was made. Major displacement of the greater tuberosity was defined as a displacement of more than $5 \mathrm{~mm}$.

Clinical examination evaluated joint active range of motion in anterior elevation, abduction, external and internal rotations. The patients' subjective assessment was recorded according to the Constant-Murley score $[5,6]$ as well as the Arm, Shoulder and Hand (DASH) score $[7,8]$.

\section{Statistical analysis:}

Statistics were calculated using SPSS 23.0 for windows. Categorical variabilities were expressed in numbers and percentages. Continuous variabilities were expressed by averages and standard deviation. Data from the two groups were compared using Pearson correlation coefficient, chi-squared test, and spearman's rank correlation. The significance level was set at $p \leq 0.05$.

\section{Results:}

Our patient pool included 8 men (26\%) and 23 women (74\%) with a mean age of 73 years, ranging from 65 to 85 . A low-energy fall was the main cause of the fractures with the dominant side being affected in $55 \%$ of cases. Four-part fractures were predominant representing $68 \%$ of the fractures ( 21 fractures). We noted 13 4-part fractures (81\%) in the group of RSA vs 8 (53\%) in the nailing group [Tab .1].

Most fractures were valgus impacted (58\%). There were 11 fractures with medial metaphyseal comminution (35.5\%)

Table 1

fracture repartition

\begin{tabular}{|llll|}
\hline \multicolumn{4}{c|}{ Fracture type } \\
\hline & 3-part & 4-part & Total \\
\hline RSA & 3 & 13 & 16 \\
\hline Anterograde nailing & 7 & 8 & 15 \\
\hline Total & 10 & 21 & 31 \\
\hline
\end{tabular}

Postoperative radiological assessment showed two unsatisfactory reductions on initial AP in the nailing group, two malunions on AP radiographic follow-up. We also reported two cases of aseptic humeral head necrosis in the 4-part fractures group. Consolidation rate was $100 \%$ with an average consolidation time of 59 days [ $45-65$ days]. The average alpha angle on AP radiographic follow-up was $47^{\circ}$. There were no cases of hardware displacement (secondary screw protrusion). 
The overall tuberosities consolidation rate was $77 \%$. Tuberosities' healing was higher in the group of RSA with an $81 \%$ rate vs $73 \%$ in the nailing group.

We observed two scapular notching (12.5\%) in the RSA group. We reported the case of a glenoid loosening that was revised with allograft.

We encountered a case of acute infection that was managed with irrigation, debridement, and antibiotic therapy. The patient had lower functional results but was satisfied on last follow-up.

The main functional results were satisfying in both groups with restauration of a functional range of motion. Main Constant and DASH scores at the last follow-up were at 68 and 24 respectively. Most patients were satisfied with the outcome of their surgeries, with an overall satisfaction rate of $87 \%$.

Functional results in the group treated with reverse total shoulder arthroplasty were better than the nailing group but with no significant differences. RSA group showed better range of motion: anterior elevation $121^{\circ}$ vs $115^{\circ}$, abduction $113^{\circ}$ vs $106^{\circ}$, external rotation $30^{\circ}$ vs $2^{\circ}$, internal rotation L3 vs L5 level. Mean functional scores for the RSA and nailing group were respectively at 74 vs 62 for the Constant score, 22 vs 26 for the DASH score.

There was no significant difference in secondary complications: $12.5 \%$ complication rate in the RSA group vs $27 \%$ in the nailing group.

Four-part fractures were associated with higher a complication rate, and lower functional results with a significant difference.

The two cases of humeral head osteonecrosis were associated with the worst functional results.

\section{Discussion:}

Surgical management of proximal humeral fractures (PHF) remains problematic with a high failure and reoperation rates. Some studies compared the functional results of ORIF and arthroplasty for the surgical management of 3- and 4- part fractures. The results found were mixed. Other studies have concluded to the presence of several factors that can predict the failure of osteosynthesis and cause a poor outcome [9].

Recognizing patient and fracture characteristics is crucial in choosing the best treatment. The vascularization status of the humeral head must be evaluated.

Age in an important factor when choosing the adequate treatment. Yahuaca et al [10] reported that the majority of patients aged $>65$ years old were treated with RSA while the other patients were more likely to undergo a conservative surgical treatment. These findings were consistent with Gupta's et al [11] in their systemic review of studies comparing ORIF, hemiarthroplasty and RSA for fractures of proximal humerus. Many studies reported worse outcomes with ORIF in this population. Hardeman et al [9] reported worse 
functional outcomes and higher re-operation rates when treating old patient with ORIF. Yahuaca et al [10] found the same results with increased non-union and reoperation rates in patients $>65$ years old. They attributed these findings to poorer bone quality in this population.

Some studies [12] evaluated the relationship between a higher ASA [13] score and the failure rate. They found that patients with higher ASA score were likely to undergo arthroplasty, and were associated with more surgical complications and prosthetic failure.

The second factor with a significant difference in the type of surgical management is Neer's fracture classification. Two-part PHF were more likely to be treated conservatively, while patients with 4-part fractures were more likely to undergo RSA. However, the optimal treatment of 3-part fractures remains difficult to determine with authors preferring RSA and others defending ORIF. The treatment is based on multiple factors like bone quality but mostly on the perfusion of the humeral head. Hertel et al [14] examined risk factors of humeral head ischemia. Predictors of ischemia were: short metaphyseal head extension $(<8 \mathrm{~mm}$ ), the disturbance of the medial hinge (displacement $>2 \mathrm{~mm}$ ) and the fracture pattern. Kloub et al [15] showed that the quality of the reduction is an important factor of determining the risk of humeral head necrosis $(\mathrm{HHN})$. HHN was observed in only $2 \%$ of fractures with good reductions, but in $60 \%$ of fractures with bad reductions.

Bastian et al [16] reported that initial ischemia after intracapsular fracture did not necessarily lead to the developpement of osteonecrosis. Vice versa, avascular necrosis may occur unexpectedly in initially perfused heads.

Avascular necrosis leads to worse functional outcomes. As soon as humeral head collapse causing screw perforation, functional scores dropped dramatically and patients suffered from pain [17].

The pattern of the fracture is also an important factor. Many series have reported poor functional outcomes of varus-impacted fractures [18]. Hardeman et al [9] reported a higher failure rate with these fractures, whereas valgus-impacted fractures result in a relatively low rate of osteonecrosis because residual vascularity may be maintained through an intact medial soft-tissue sleeve [19]. Varus-impacted fractures are more likely to disrupt this sleeve and more likely to be instable.

Reoperation rate is an important factor when deciding the appropriate management for PHF. Gupta et al [11] reported a higher reoperation rate of $12.7 \%$ in ORIF vs $5 \%$ in RSA. Yahuaca et al [10] noted the same results with $17.1 \%$ in ORIF vs $6.6 \%$ in RSA.

The elderly patients are a fragile population with a high level of postoperative morbidity and mortality rates. This needs to be considered when making the treatment decision.

Many authors have analyzed predictive factors of failure in ORIF in order to assist the surgeon in the decision-making process in managing these difficult fractures. A significantly displaced, poorly vascularized varus articular fracture in the older patient predicts a poor outcome [9]. Thus, we think this is the ideal indication of RSA. 
Our study has its limitations. Most are related to inherent weakness of a retrospective review, including loss to follow-up. Our study had a small population and a low follow-up making the evaluation of some long-term complications hard.

\section{Conclusion:}

Proximal humerus fractures are prevalent and widespread. Surgical management remains problematic with high failure and re-operation rates. Analyzing the patient and fracture characteristics is very important in order to choose the correct treatment.

Reverse total shoulder arthroplasty has become an interesting surgical alternative, as the functional and anatomical results in our study were promising, while elucidating an improved quality of life for our patients. These findings have encouraged us to continue using this technique as a first-line choice for complex fractures of the proximal humerus in the elderly.

\section{Abbreviations:}

AP: anteroposterior

DASH: Arm, Shoulder and Hand

HHN: humeral head necrosis

ORIF: open reduction internal fixation

PHF: proximal humeral fractures

RSA: reverse shoulder arthroplasty

\section{Declarations:}

\section{Ethics Approval and consent to participate}

As per university standard guideline, participant consent and ethical approval have been collected and preserved by the authors.

\section{Consent for Publication}

Participant consent and ethical approval have been collected and preserved by the authors.

\section{Availability of supporting data}

The data supporting our findings is available and can be found in the orthopedics department of the Military Hospital of Tunis. 


\section{COMPETING INTERESTS}

Authors have declared that no competing interests exist.

\section{Source of funding}

No external funding was received for this study.

\section{Authors' contributions}

All authors contributed to the elaboration of this study. Khalil AMRI and Rabie AYARI designed the study, performed the statistical analysis, wrote the protocol, and wrote the first draft of the manuscript. Achraf ABDENNADHER, Mohamed BEN SALAH and Rim DHAHRI managed the analyses of the study. Chadia CHOURABI performed cardiologic assessment of our patients. Karim LATRECH TLEMSANI and Lotfi NOUISRI managed the literature searches.

All authors read and approved the final manuscript.

\section{Acknowledgements}

Not applicable

\section{References:}

1. Kannus P, Palvanen M, Niemi S, Parkkari J, Järvinen M, Vuori I. Increasing number and incidence of osteoporotic fractures of the proximal humerus in elderly people. BMJ. 1996;313(7064):1051-2.

2. Kannus P, Palvanen M, Niemi S, Parkkari J, Järvinen M, Vuori I. Osteoporotic fractures of the proximal humerus in elderly Finnish persons: sharp increase in 1970-1998 and alarming projections for the new millennium. Acta Orthop Scand. 2000;71(5):465-70.

3. Sperling JW, Cuomo F, Hill JD, Hertel R, Chuinard C, Boileau P. The difficult proximal humerus fracture: tips and techniques to avoid complications and improve results. Instr Course Lect. 2007;56:45-57.

4. Neer CSI. Displaced Proximal Humeral Fractures: PART I. Classification and evaluation. JBJS. 1970;52(6):1077-1089.

5. Constant CR, Murley AH. A clinical method of functional assessment of the shoulder. Clin Orthop Relat Res. 1987;(214):160-4.

6. Constant CR, Gerber C, Emery RJH, Søjbjerg JO, Gohlke F, Boileau P. A review of the Constant score: modifications and guidelines for its use. J Shoulder Elbow Surg. 2008;17(2):355-61.

7. Gummesson C, Atroshi I, Ekdahl C. The disabilities of the arm, shoulder and hand (DASH) outcome questionnaire: longitudinal construct validity and measuring self-rated health change after surgery. BMC Musculoskelet Disord. 2003;4:11. 
8. Hudak PL, Amadio PC, Bombardier C. Development of an upper extremity outcome measure: the DASH (disabilities of the arm, shoulder and hand). The Upper Extremity Collaborative Group (UECG). Am J Ind Med. 1996;29(6):602-8.

9. Hardeman F, Bollars P, Donnelly M, Bellemans J, Nijs S. Predictive factors for functional outcome and failure in angular stable osteosynthesis of the proximal humerus. Injury. 2012;43(2):153-8.

10. Yahuaca BI, Simon P, Christmas KN, Patel S, Gorman RA, Mighell MA, et al. Acute surgical management of proximal humerus fractures: ORIF vs. hemiarthroplasty vs. reverse shoulder arthroplasty. J Shoulder Elbow Surg. 2020;1-9.

11. Gupta AK, Harris JD, Erickson BJ, Abrams GD, Bruce B, McCormick F, et al. Surgical management of complex proximal humerus fractures-a systematic review of 92 studies including 4500 patients. $J$ Orthop Trauma. 2015;29(1):54-9.

12. Johnson CC, Sodha S, Garzon-Muvdi J, Petersen SA, McFarland EG. Does preoperative American Society of Anesthesiologists score relate to complications after total shoulder arthroplasty? Clin Orthop Relat Res. 2014;472(5):1589-96.

13. Owens WD, Felts JA, Spitznagel EL. ASA physical status classifications: a study of consistency of ratings. Anesthesiology. 1978;49(4):239-43.

14. Hertel R, Hempfing A, Stiehler M, Leunig M. Predictors of humeral head ischemia after intracapsular fracture of the proximal humerus. J Shoulder Elbow Surg. 2004;13(4):427-33.

15. Kloub M, Holub K, Urban J, Látal P, Peml M, Křivohlávek M. Intramedullary nailing of displaced fourpart fractures of the proximal humerus. Injury. 2019;50(11):1978-85.

16. Bastian JD, Hertel R. Initial post-fracture humeral head ischemia does not predict development of necrosis. J Shoulder Elbow Surg. 2008;17(1):2-8.

17. Zirngibl B, Biber R, Bail HJ. Humeral head necrosis after proximal humeral nailing: what are the reasons for bad outcomes? Injury. 2016;47 Suppl 7 :S10-3.

18. Aggarwal S, Bali K, Dhillon MS, Kumar V, Mootha AK. Displaced proximal humeral fractures: an Indian experience with locking plates. J Orthop Surg Res. 2010;5:60.

19. Lavender A, Srinivasan M, Henderson A. Percutaneous reduction and fixation of the valgus impacted fracture of the proximal humerus: a new method. J Bone Joint Surg Br. 1998;80(1):105. 